\title{
REVIEW
}

\section{Role of thyroglobulin on negative feedback autoregulation of thyroid follicular function and growth}

\author{
Koichi Suzuki, Akira Kawashima, Aya Yoshihara, Takeshi Akama, Mariko Sue, Akio Yoshida ${ }^{1}$ \\ and Hiroaki J Kimura² \\ Laboratory of Molecular Diagnostics, Department of Mycobacteriology, National Institute of Infectious Diseases, Leprosy Research Center, 4-2-1 Aoba-cho, \\ Higashimurayama, Tokyo 189-0002, Japan \\ ${ }^{1}$ Division of Regenerative Medicine and Therapeutics, Department of Genetics and Regenerative Medicine, Tottori University Graduate School of Medicine, \\ Tottori 683-8504, Japan \\ ${ }^{2}$ Division of Bioimaging Sciences, Center for Molecular Medicine, Jichi Medical University, Shimotsuke 329-0498, Japan \\ (Correspondence should be addressed to K Suzuki; Email: koichis@nih.go.jp)
}

\begin{abstract}
Thyroid function is tightly regulated by TSH. Although individual follicles are exposed to the same blood supply of TSH and express relatively homogenous levels of the TSH receptor, the function of individual follicles is variable. It was shown that thyroglobulin $(\mathrm{Tg})$, stored in the follicular lumen, is a potent negative feedback regulator of follicular function. Thus, physiological concentrations of $\mathrm{Tg}$ significantly suppress thyroid-specific gene expression and antagonize the
\end{abstract}

TSH-mediated stimulation that induces expression of thyroid-specific genes. Tg coordinately regulates both basal and apical iodide transporters in thyroid follicular cells. Recently, it was also reported that $\mathrm{Tg}$ could induce thyroid cell growth in the absence of TSH. These results indicate that $\mathrm{Tg}$ is an essential autocrine regulator of physiological thyroid follicular function that counteracts the effects of TSH.

Journal of Endocrinology (2011) 209, 169-174

\section{Introduction}

Serum levels of thyroid hormone are tightly regulated by the levels of TSH in serum. However, TSH is not the only factor regulating thyroid function; it is also regulated by various other factors, including iodine and autoantibodies. Here, we show that the thyroglobulin $(\mathrm{Tg})$ stored in thyroid follicles (the most abundant product of thyroid cells) functions as a potent negative feedback autocrine regulator of endocrine function and cell growth in individual follicles. We also discuss potential pathological conditions that may occur in human patients due to irregularities in Tg-mediated regulation of thyroid function.

\section{Follicular function and heterogeneity of the thyroid}

The thyroid follicle is the most unique structure of the thyroid gland; the thyroid follicle is where iodine is trapped, $\mathrm{Tg}$ is stored, and thyroid hormones are formed and stored. The function of the entire thyroid gland reflects the sum of the function of each follicle (Dunn \& Dunn 2000). In some marine fish, such as lamprey, $\mathrm{Tg}$ and thyroid hormones are synthesized and secreted by specialized cells in the endostyle, and there is no follicular structure (Wright et al. 1978, Fredriksson et al. 1988). Therefore, the large follicular space found in many other animals, including all mammals, may be required to efficiently utilize dietary iodide and store the thyroid hormone and its precursors until needed.

TSH stimulates iodide uptake from the blood stream and the synthesis of $\mathrm{Tg}$, thyroid peroxidase (TPO), and the sodium/iodide symporter (NIS), in order to iodinate $\mathrm{Tg}$ to form thyroid hormones. After $\mathrm{Tg}$ is synthesized, it is transported to the apical membrane and released into the follicular lumen (Dunn \& Dunn 2000). TPO and $\mathrm{H}_{2} \mathrm{O}_{2}$ at the apical membrane catalyze the iodination of tyrosine residues on $\mathrm{Tg}$ and then couple two iodotyrosine residues to form thyroxine $\left(\mathrm{T}_{4}\right)$ and triiodothyronine $\left(\mathrm{T}_{3}\right)$. TSH stimulates the reabsorption and hydrolysis of follicular $\mathrm{Tg}$ and the subsequent secretion of thyroid hormones in the blood. Iodinated $\mathrm{Tg}$ is reabsorbed back into follicular cells through the apical membrane and degraded to form $T_{3} / T_{4}$ in lysosomes. The $T_{3} / T_{4}$ is then secreted through the basal membrane (Dunn \& Dunn 2000).

TSH is supplied to thyrocytes by a fine capillary network that surrounds each follicle. Serum TSH levels are fairly 
constant, and the expression of the TSH receptor (TSHR) along the basolateral membrane of follicular cells is largely homogeneous. Despite the fact that each follicle has a uniform TSH supply and reasonably uniform TSHR expression, the function of individual follicles is not uniform, but rather heterogeneous. Follicles are quite heterogeneous in their morphology, i.e. shape and size (or colloid density) and in their function, i.e. iodide uptake, diffusion of iodinated $\mathrm{Tg}$ within the follicular lumen, and accumulation of $\mathrm{Tg}$ and thyroid hormones (Gerber et al. 1985, 1986, Suzuki et al. 1999a,b,c).

There are several possibilities that could explain the nature of follicular heterogeneity. Some evidence suggests that individual thyrocytes within the follicle are not functionally uniform. Using chimeric mice, it has been shown that epithelial cells surrounding a follicle do not originate from a single cell, but are mosaic (Feder 1976, Thomas et al. 1989). Using a three-dimensional collagengel culture method, it was shown that cell aggregates formed follicular structures (Toda et al. 1993). Primary cultured thyrocytes showed quite heterogeneous behaviors (Baptist et al. 1991), and it is known that cloned thyroid cells show significant variability in growth and function in culture (Huber et al. 1990). These findings suggest that thyrocytes surrounding a single follicle are heterogeneous in origin and that the function of each follicle is also heterogeneous, such that the heterogeneity cannot be explained solely by differences in the composition of follicular cells within the follicle. Until recently, the true nature of such follicular heterogeneity was unclear.

\section{Negative feedback autoregulation of thyroid-specific gene expression and follicular function by $\mathrm{Tg}$}

Using cultured rat thyroid FRTL-5 cells, we have shown that mRNA levels of Tg, TPO, and NIS were significantly suppressed by physiological concentrations of follicular $\mathrm{Tg}$ added to the culture medium (Suzuki et al. 1998, 1999d). The $\mathrm{Tg}$ action was concentration-dependent, and the same concentrations of BSA, immunoglobulin, or mannitol had no effect. Inorganic iodide, $\mathrm{T}_{3}$, or $\mathrm{T}_{4}$ did not affect $\mathrm{Tg}$ mRNA levels either. In contrast to the suppression of thyroid-specific gene expression, the mRNA levels of the Pendred syndrome gene (PDS) and major histocompatibility complex (MHC) class I gene were increased by low concentrations of $\mathrm{Tg}$ (Suzuki et al. 1998, Royaux et al. 2000). Actually, Tg is the only factor that can induce PDS gene expression in the thyroid (Royaux et al. 2000).

$\mathrm{Tg}$ suppresses radioiodine uptake in FRTL-5 cells in a dose- and time-dependent manner (Suzuki et al. 1999d). Although iodide accumulation in rat thyroid follicles is quite heterogeneous (Wollman \& Loewenstein 1973, Suzuki et al. 1999a,c), in vivo studies further indicate that follicular Tg is a negative feedback regulator of iodide uptake in each follicle.
For example, in a study of propylthiouracil-treated rats, we showed that a rim of $\mathrm{Tg}$ that bound to the apical membrane in the follicular lumen was associated with the suppression of thyroid transcription factor (TTF-1) mRNA and Tg biosynthesis (Suzuki et al. 1999b). It was further demonstrated that there was a clear inverse correlation between $\mathrm{Tg}$ accumulation and radioiodine uptake in each follicle (Suzuki et al. 1999d).

The range of $\mathrm{Tg}$ concentrations required for these effects was $1-10 \mathrm{mg} / \mathrm{ml}$, which is within the range of normal follicular $\mathrm{Tg}$ concentrations, $0 \cdot 1$ up to $250 \mathrm{mg} / \mathrm{ml}$, as measured by aspiration biopsy or micropuncture of a single follicle (Hayden et al. 1970, Smeds 1972, Salabe et al. 1996). The relatively large range of the estimates of follicular $\mathrm{Tg}$ concentration may be the result of significant functional heterogeneity among follicles. Tg concentrations of $0.1 \mathrm{mg} / \mathrm{ml}$, which is still much higher than serum $\mathrm{Tg}$ concentration, had a minimal effect. Thus, we believe that $\mathrm{Tg}$ acts through the apical, not the basal membrane, of the follicular epithelium.

Changes in mRNA levels seemed to be regulated transcriptionally because the promoter activity of $\mathrm{Tg}$, TPO, and NIS decreased with the decreases in mRNA levels (Suzuki et al. 1998, 1999d). In fact, follicular Tg significantly decreased mRNA levels of three thyroid-specific transcription factors, TTF-1, TTF-2, and Pax- 8 , but not ubiquitous factors, Sox-4, TSHR suppressor element-binding protein-1 (TSEP-1), and single-strand binding protein-1 (SSBP-1). Consistent with the suppression of mRNA levels of thyroidspecific transcription factors, $\mathrm{Tg}$ also decreased TTF-1, TTF-2, and Pax-8 binding to their specific DNA-binding sites on the Tg and TSHR promoters (Suzuki et al. 1998). Additionally, the promoter activity of the $5^{\prime}$-flanking region of a TTF-1-luciferase reporter gene chimera was decreased by $\mathrm{Tg}$ in a concentration-dependent manner (Suzuki et al. 1998, Nakazato et al. 2000). These results indicate that $\mathrm{Tg}$ protein suppresses expression of $\mathrm{Tg}$, TPO, and NIS by suppressing multiple thyroid-specific transcription factors. Therefore, we suggest that $\mathrm{Tg}$ protein, accumulated within the follicle, acts as a negative feedback regulator and contributes to the follicular heterogeneity.

\section{$\mathrm{Tg}$ induces growth of thyrocytes in the absence of TSH}

The growth of thyroid cells is tightly regulated by the coordinated action of TSH and insulin/insulin-like growth factor 1 (IGF1) signaling (Takahashi et al. 1991, Nedachi et al. 2000). However, we have shown that $\mathrm{Tg}$ alone can stimulate cell growth and ${ }^{3} \mathrm{H}-\mathrm{TdR}$ uptake in FRTL-5 cells as effectively as the combination of TSH, insulin, and serum (Noguchi et al. 2010). Although Tg stimulated cell growth, it did not increase cAMP levels in FRTL-5 cells, and an inhibitor of cAMPdependent protein kinase, $\mathrm{H}-89$, had no effect on Tg-induced cell growth. Moreover, TSH/cAMP counteracted the ability 
of $\mathrm{Tg}$ to induce cell growth. Therefore, the intracellular signaling utilized by $\mathrm{Tg}$ appears to be quite different from that used by TSH.

Both Tg and insulin/IGF1 activated AKT, a key downstream molecule that mediates insulin/IGF1 action, and a phosphatidylinositol 3-kinase (PI3K) inhibitor, LY294002, significantly suppressed $\mathrm{Tg}$-induced ${ }^{3} \mathrm{H}-\mathrm{TdR}$ incorporation (Noguchi et al. 2010). The effect of $\mathrm{Tg}$ and insulin in stimulating cell growth was additive, which suggests that $\mathrm{Tg}$ may utilize PI3K/AKT to induce cell growth, but possibly by a mechanism that involves a signaling cascade that is not yet known to function in this process.

Lower concentrations of $\mathrm{Tg}(1-5 \mathrm{mg} / \mathrm{ml})$ induce more cell growth than higher concentrations $(>10 \mathrm{mg} / \mathrm{ml})$. All these $\mathrm{Tg}$ concentrations are within the lower range of follicular $\mathrm{Tg}$ in different follicles of the normal thyroid (Hayden et al. 1970, Smeds 1972, Salabe et al. 1996). Tg-mediated suppression of thyroid-specific gene expression is dose-dependent (Suzuki et al. 1998, 1999d, Suzuki \& Kohn 2006, Noguchi et al. 2010); however, Tg induced expression of at least two genes, MHC class I and PDS (Suzuki et al. 1998, Royaux et al. 2000, Suzuki \& Kohn 2006). Thus, mRNA levels of MHC class I and PDS are highest at lower concentrations of $\mathrm{Tg}$ and decrease with higher Tg concentrations (Suzuki et al. 1998, Royaux et al. 2000, Suzuki \& Kohn 2006, Noguchi et al. 2010). Therefore, optimal $\mathrm{Tg}$ concentrations that induce growth promotion and suppression of gene expression showed biphasic pattern.

It is interesting that the 'stimulation' of thyroid cells, i.e. the induction of cell growth and gene expression of MHC class I and PDS - is higher with lower concentrations of Tg, while the 'suppression' of gene expression and follicular function is induced by higher concentrations of $\mathrm{Tg}$ in a dose-dependent manner. These results suggest that there are at least two distinct mechanisms of $\mathrm{Tg}$ action that have different kinetics. Both effects, 'stimulation' and 'suppression', increase in the order of $27 \mathrm{~S}>19 \mathrm{~S}>12 \mathrm{~S} \mathrm{Tg}$. Thus, using gel-fractionated $\mathrm{Tg}$ moieties, it was demonstrated that $27 \mathrm{~S} \mathrm{Tg}$ has the strongest effect on induction of cell growth (Noguchi et al. 2010) and suppression of TTF-1 expression (Suzuki et al. 1999a,c, Ulianich et al. 1999). Therefore, it is possible that different recognition systems, rather than the two different domains of $\mathrm{Tg}$ protein, are responsible for the biphasic action.

An important question is how such a large molecule could exert its regulatory action under physiological conditions at the transcriptional level. Several Tg-binding proteins have been described and are thought to be involved in the post-translational modification of $\mathrm{Tg}$ or in a cyclical micropinocytotic process: an $\mathrm{N}$-acetylglucosamine receptor (Miquelis et al. 1987), an asialoglycoprotein receptor (ASGPR; Consiglio et al. 1979), and gp330/megalin (Zheng et al. 1998). Unfortunately, the Tg-recognition system and the detailed signaling pathways downstream of Tg remain largely unknown.

\section{Physiological cycle of follicular activity regulated by $\mathrm{Tg}$}

TSH primarily affects endocytosis, hydrolysis, and secretion of hormones rather than activating gene expression or initiating synthesis and storage of Tg. Resorption of follicular colloid occurs within a short time after TSH stimulation, whereas transcription, synthesis, and secretion of newly formed $\mathrm{Tg}$ are quantitatively limited; therefore, the storage space, i.e. the follicular lumen - refills over a longer time frame (Yi et al. 1997, Suzuki et al. 1999a,c). This delay is evident in morphological observations after TSH stimulation in which follicles have less colloid and collapsed follicular lumen, while follicles with low TSH stimulation are filled with dense colloid, rich with $\mathrm{Tg}$ and thyroid hormones (Wollman \& Loewenstein 1973, Yi et al. 1997, Suzuki et al. 1999b). These differences can be readily understood, as resorption and degradation are relatively simple physical and chemical reactions, whereas transcription, translation, and post-translational modification require many other proteins and steps, and therefore, considerably more time and energy. These observations indicate that, although TSH stimulates gene expression of Tg, TPO, and NIS, this TSH-mediated stimulation of gene expression is not enough to refill the follicular stores that were absorbed by follicular cells following TSH action. As a result, the volume of reabsorbed follicular colloid is much larger than the volume resulting from synthesis and secretion through transcription, translation, and protein modification.

Some follicles store a large amount of $\mathrm{Tg}$ and thyroid hormones, while others store much less (Suzuki et al. $1999 a, b)$. Tg seems to be the main regulator of thyroidspecific gene expression in individual follicles under relatively constant serum TSH level; therefore, we hypothesize that each follicle has its own synthesis and secretion cycle, depending on the amount of $\mathrm{Tg}$ stored in the follicle. We suggest that this cycle is regulated in part by a balance between maximal Tg suppression of gene expression and TSHregulated resorption of the colloid. TSH increases resorption of the follicular colloid, decreases Tg storage, and releases Tg suppression, thereby allowing TSH-mediated stimulation of gene expression to become dominant (Suzuki et al. 1999a,b,c,d, Nakazato et al. 2000, Kohn et al. 2001, Suzuki \& Kohn 2006).

In a follicle where large amounts of $\mathrm{Tg}$ has accumulated, gene expression is suppressed (Suzuki et al. 1998, 1999d); therefore, new $\mathrm{Tg}$ biosynthesis is low (Fig. 1). In this situation, TSH will act predominantly on resorption of the colloid. On the other hand, in a follicle where $\mathrm{Tg}$ concentration is low, negative feedback regulation of $\mathrm{Tg}$ is weak, and suppression of gene expression is minimal (Fig. 1). Consequently, TSH can fully activate gene expression and $\mathrm{Tg}$ biosynthesis increases. Efficiency of resorption and degradation of follicular Tg will be low because of the low levels of stored Tg in such a follicle. When Tg concentrations 


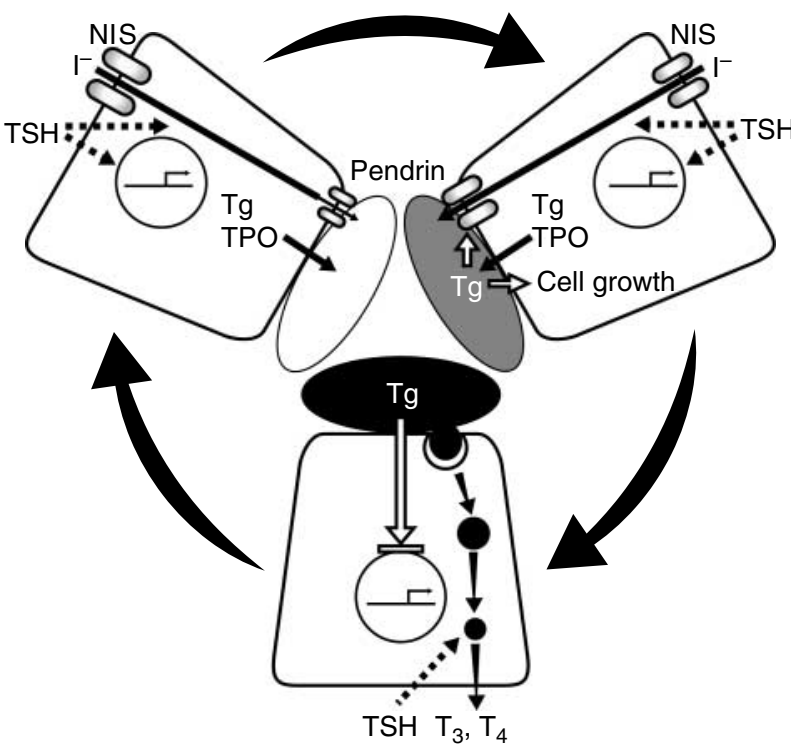

Figure $1 \mathrm{~A}$ revised model for the physiological regulation of gene expression, follicular function and cell growth by coordinate regulation of follicular Tg content and serum TSH. In a follicle where $\mathrm{Tg}$ accumulation is minimal, thyroid-specific gene expression is at a maximum (left). When a follicle accumulates a low concentration of $\mathrm{Tg}$, maximal PDS gene expression is induced, and iodide efflux reaches a maximal (right). When sufficient Tg accumulates within a follicle (middle), all gene expression necessary for iodide transport and hormone biosynthesis is suppressed. When accumulated follicular Tg decreases to a certain level, Tg suppression of gene expression ceases, and the whole process could repeat.

in the follicular lumen gradually increase with new synthesis, cell growth and pendrin expression are maximally induced (Royaux et al. 2000, Suzuki \& Kohn 2006, Noguchi et al. 2010). These conditions allow efficient transportation of iodide into the follicular lumen and expand the size of the follicle in order to synthesize and store thyroid hormone precursors. When enough Tg accumulates in the follicle, the processes of gene expression, iodide transportation, and cell growth are coordinately suppressed. The follicle with full $\mathrm{Tg}$ is ready to be reabsorbed and to secrete thyroid hormones. During the course of decreasing follicular Tg content due to reabsorption, the follicle is supposed to shrink. Although it will be difficult to assess this process in vivo, this mechanism will limit follicular size and, therefore, suppress the development of goiters under normal conditions.

One question that remains is whether $\mathrm{Tg}$ affects the processes of resorption and degradation of the follicular colloid. It is likely that the processes of resorption and degradation of follicular colloid occur differently in follicles with different levels of Tg. If colloid droplets are absorbed into thyrocytes from follicles with low Tg concentration, the efficiency of thyroid hormone generation is very low. Therefore, it would be reasonable to predict a mechanism in which follicular $\mathrm{Tg}$ stimulates the resorption/degradation pathway and the regulation of gene expression.

\section{Pathologic consequences}

We suggest the following pathologic consequences, which might be related to abnormalities of $\mathrm{Tg}$-mediated regulatory mechanism and to failure of physiological regulation of follicular function. In cases where $\mathrm{Tg}$ suppression is weakened, either by an abnormality of $\mathrm{Tg}$ itself or aberrations in the mechanism by which $\mathrm{Tg}$ suppresses gene transcription, constant activation of gene expression may occur, resulting in persistent cell growth or accumulation of colloid and enlargement of follicles. This condition might be related to some form of goiter. In cases where $\mathrm{Tg}$ suppression is enhanced, gene expression is constantly reduced, which may result in hypothyroidisms and/or compensatory enlargement of follicles. When MHC class I expression is increased by $\mathrm{Tg}$, inflammatory cell infiltration and immune reaction may be induced. Similarly, although the molecular mechanisms underlying Tg-induced growth stimulation are not known, an imbalance or a dysregulation of such a mechanism could relate to aberrant thyroid cell growth. Some of the thyroproliferative disorders with unknown etiology might be due to aberrations in Tg-regulated cell growth.

We have previously shown that $\mathrm{Tg}$ prepared from iodidedeficient goiters, colloid adenomas or congenital goiters suppresses thyroid-specific gene expression more effectively than does the Tg prepared from normal tissue (Suzuki et al. 1998, 1999d). Therefore, poorly iodinated and/or poorly sialylated Tg may induce thyroid cell growth more effectively than fully iodinated and/or sialylated Tg. This possibility is consistent with previous observations that inhibition of iodide coupling is influenced by the iodination and sialylation of the Tg molecule (Studer et al. 1986). It is also consistent with the observation that poorly iodinated $\mathrm{Tg}$ in nontoxic, multinodular sporadic goiters inhibits TSH-induced Tg utilization from the follicular lumen (Sinadinovic et al. 1978).

$\mathrm{Tg}$ has strong physiological effects that regulate follicular function and thyroid cell growth. Therefore, abnormality in $\mathrm{Tg}$ itself or in a Tg-mediated mechanism may result in pathological conditions in human patients. Although, it is not currently known whether any thyroid disorder is caused by one of these abnormalities, understanding of cellular recognition system of $\mathrm{Tg}$ and signaling cascades will lead to a further understanding of potential pathological consequences of these abnormalities.

\section{Conclusion}

Follicular $\mathrm{Tg}$, a thyroid hormone precursor, is a potent suppressor of thyroid-specific gene transcription and stimulator of thyroid cell growth. Low levels of Tg in the follicular lumen stimulate cell growth and iodide transport to accelerate the iodide organification process, but elevated $\mathrm{Tg}$ levels in the follicle shut down all of these functions. This novel mechanism may be a major autocrine pathway within the 
thyroid follicle and may serve as a compensatory function that maintains follicular homeostasis. Furthermore, this negative feedback mechanism may contribute to the heterogeneity of the size and function of individual follicles in the same gland, i.e. regulating follicular function, controlling the amount of colloid within the follicle, and limiting the size and cell growth of each follicle. Studies of the molecular mechanism of $\mathrm{Tg}$ action are needed to gain a better understanding of thyroid physiology and pathology.

Fundamental questions remain regarding which part of the Tg molecule is recognized by which mechanism (receptors) and which intracellular signals transduce the Tg signal and elicit these strong physiological effects. To address these questions, we are currently studying the physiological role of a short $\mathrm{Tg}$ variant $(\mathrm{kTg})$ that we have recently cloned from kidney glomerulus (Wu et al. 2009). We are also analyzing changes in gene expression following $\mathrm{Tg}$ stimulation using DNA microarrays. Other efforts are also underway to address some of these concerns.

\section{Declaration of interest}

The authors declare that there is no conflict of interest that could be perceived as prejudicing the impartiality of the review reported.

\section{Funding}

This work was supported in part by a Grant-in-Aid for Scientific Research from the Japan Society for the Promotion of Science (\#21591187 to K S) and Thyroid Basic Research Award and Cosmic Research Innovation Award, both from Japan Thyroid Association (both to K S).

\section{Acknowledgements}

The authors thank Dr Leonard D Kohn, Dr Akira Kawaoi, and Dr Ryohei Katoh for their contribution to this study.

\section{References}

Baptist M, Pohl V, Dumont JE \& Roger PP 1991 Various facets of the intercellular heterogeneity in thyroid primary culture. Thyroidology 3 109-113.

Consiglio E, Salvatore G, Rall JE \& Kohn LD 1979 Thyroglobulin interactions with thyroid plasma membranes. The existence of specific receptors and their potential role. Journal of Biological Chemistry 254 5065-5076.

Dunn JT \& Dunn AD 2000 Thyroglobulin: chemistry, biosynthesis, and proteolysis. In Werner \& Ingbar's The Thyroid: A Fundamental and Clinical Text, 8th edn, pp 91-104. Eds LE Braverman \& RD Utiger. Philadelphia, PA: Lippincott Williams \& Wilkins.

Feder N 1976 Solitary cells and enzyme exchange in tetraparental mice. Nature 263 67-69. (doi:10.1038/263067a0)

Fredriksson G, Ofverholm T \& Ericson LE 1988 Iodine binding and peroxidase activity in the endostyle of Salpa fusiformis, Thalia democratica, Dolioletta gegenbauri and Doliolum nationalis (Tunicata, Thaliacea). Cell and Tissue Research 253 403-411. (doi:10.1007/BF00222297)
Gerber H, Studer H \& von Grunigen C 1985 Paradoxical effects of thyrotropin on diffusion of thyroglobulin in the colloid of rat thyroid follicles after long term thyroxine treatment. Endocrinology 116 303-310. (doi:10.1210/endo-116-1-303)

Gerber H, Peter HJ \& Studer H 1986 Diffusion of thyroglobulin in the follicular colloid. Endocrinologia Experimentalis 20 23-33.

Hayden LJ, Shagrin JM \& Young JA 1970 Micropuncture investigation of the anion content of colloid from single rat thyroid follicles. A micromethod for the simultaneous determination of iodide and chloride in nanomole quantities. Pflügers Archiv 321 173-186. (doi:10.1007/BF00586371)

Huber G, Derwahl M, Kaempf J, Peter HJ, Gerber H \& Studer H 1990 Generation of intercellular heterogeneity of growth and function in cloned rat thyroid cells (FRTL-5). Endocrinology 126 1639-1645. (doi:10.1210/ endo-126-3-1639)

Kohn LD, Suzuki K, Nakazato M, Royaux I \& Green ED 2001 Effects of thyroglobulin and pendrin on iodide flux through the thyrocyte. Trends in Endocrinology and Metabolism 12 10-16. (doi:10.1016/S1043$2760(00) 00337-4)$

Miquelis R, Alquier C \& Monsigny M 1987 The N-acetylglucosaminespecific receptor of the thyroid, binding characteristics, partial characterization, and potential role. Journal of Biological Chemistry 262 15291-15298.

Nakazato M, Chung HK, Ulianich L, Grassadonia A, Suzuki K \& Kohn LD 2000 Thyroglobulin repression of thyroid transcription factor 1 (TTF-1) gene expression is mediated by decreased DNA binding of nuclear factor I proteins which control constitutive TTF-1 expression. Molecular and Cellular Biology 20 8499-8512. (doi:10.1128/MCB.20.22. 8499-8512.2000)

Nedachi T, Akahori M, Ariga M, Sakamoto H, Suzuki N, Umesaki K, Hakuno F \& Takahashi SI 2000 Tyrosine kinase and phosphatidylinositol 3 -kinase activation are required for cyclic adenosine $3^{\prime}, 5^{\prime}$-monophosphatedependent potentiation of deoxyribonucleic acid synthesis induced by insulin-like growth factor-I in FRTL-5 cells. Endocrinology 141 2429-2438. (doi:10.1210/en.141.7.2429)

Noguchi Y, Harii N, Giuliani C, Tatsuno I, Suzuki K \& Kohn LD 2010 Thyroglobulin $(\mathrm{Tg})$ induces thyroid cell growth in a concentration-specific manner by a mechanism other than thyrotropin/cAMP stimulation. Biochemical and Biophysical Research Communications 391 890-894. (doi:10. 1016/j.bbrc.2009.11.158)

Royaux IE, Suzuki K, Mori A, Katoh R, Everett LA, Kohn LD \& Green ED 2000 Pendrin, the protein encoded by the Pendred syndrome gene (PDS), is an apical porter of iodide in the thyroid and is regulated by thyroglobulin in FRTL-5 cells. Endocrinology 141 839-845. (doi:10.1210/en.141.2.839)

Salabe GB, Corvo L \& Lotz H 1996 Thyroglobulin determined in thyroid fine needle aspiration biopsies by radial immunodiffusion and electroimmunodiffusion. European Journal of Clinical Chemistry and Clinical Biochemistry 34 43-47. (doi:10.1515/cclm.1996.34.1.43)

Sinadinovic J, Kraincanic M, Micic JV, Kostic G \& Matic G 1978 Some disturbances related to iodination and utilisation of thyroglobulin and $27 \mathrm{~S}$ iodoprotein in non-toxic multinodular goitre. Endokrinologie 72 155-165.

Smeds SA 1972 A microgel electrophoretic analysis of the colloid proteins in single rat thyroid follicles. I. The qualitative protein composition of the colloid in normal thyroids. Endocrinology 91 1288-1299. (doi:10.1210/ endo-91-5-1288)

Studer H, von Grunigen C, Haeberli A, Kohler H, Rothlisberger M \& Gerber H 1986 Iodination of thyroglobulin molecules depends on their diffusion velocity in follicular colloid. Molecular and Cellular Endocrinology 45 91-103. (doi:10.1016/0303-7207(86)90136-X)

Suzuki K \& Kohn LD 2006 Differential regulation of apical and basal iodide transporters in the thyroid by thyroglobulin. Journal of Endocrinology 189 247-255. (doi:10.1677/joe.1.06677)

Suzuki K, Lavaroni S, Mori A, Ohta M, Saito J, Pietrarelli M, Singer DS, Kimura S, Katoh R, Kawaoi A et al. 1998 Autoregulation of thyroidspecific gene transcription by thyroglobulin. PNAS 95 8251-8256. (doi:10. 1073/pnas.95.14.8251)

Suzuki K, Lavaroni S, Mori A, Yamamoto K, Yi X, Miyagi E, Katoh R, Kohn LD \& Kawaoi A 1999a Thyroglobulin: a master regulator of follicular function via transcriptional suppression of thyroid specific genes. Acta Histochemica et Cytochemica 32 111-119. 
Suzuki K, Mori A, Lavaroni S, Miyagi E, Ulianich L, Katoh R, Kawaoi A \& Kohn LD 1999 b In vivo expression of thyroid transcription factor-1 RNA and its relation to thyroid function and follicular heterogeneity: identification of follicular thyroglobulin as a feedback suppressor of thyroid transcription factor-1 RNA levels and thyroglobulin synthesis. Thyroid 9 319-331. (doi:10.1089/thy.1999.9.319)

Suzuki K, Mori A, Lavaroni S, Ulianich L, Miyagi E, Saito J, Nakazato M, Pietrarelli M, Shafran N, Grassadonia A et al. 1999c Thyroglobulin regulates follicular function and heterogeneity by suppressing thyroid-specific gene expression. Biochimie 81 329-340. (doi:10.1016/ S0300-9084(99)80078-9)

Suzuki K, Mori A, Saito J, Moriyama E, Ullianich L \& Kohn LD 1999d Follicular thyroglobulin suppresses iodide uptake by suppressing expression of the sodium/iodide symporter gene. Endocrinology 140 5422-5430. (doi:10.1210/en.140.11.5422)

Takahashi S, Conti M, Prokop C, Van Wyk JJ \& Earp HS III 1991 Thyrotropin and insulin-like growth factor I regulation of tyrosine phosphorylation in FRTL-5 cells. Interaction between cAMP-dependent and growth factor-dependent signal transduction. Journal of Biological Chemistry 266 7834-7841.

Thomas GA, Williams D \& Williams ED 1989 The clonal origin of thyroid nodules and adenomas. American Journal of Pathology 134 141-147.

Toda S, Yonemitsu N, Minami Y \& Sugihara H 1993 Plural cells organize thyroid follicles through aggregation and linkage in collagen gel culture of porcine follicle cells. Endocrinology 133 914-920. (doi:10.1210/en.133.2. 914)

Ulianich L, Suzuki K, Mori A, Nakazato M, Pietrarelli M, Goldsmith P, Pacifico F, Consiglio E, Formisano S \& Kohn LD 1999 Follicular thyroglobulin (TG) suppression of thyroid-restricted genes involves the apical membrane asialoglycoprotein receptor and TG phosphorylation. Journal of Biological Chemistry 274 25099-25107. (doi:10.1074/jbc.274.35 25099)

Wollman SH \& Loewenstein JE 1973 Rates of colloid droplet and apical vesicle production and membrane turnover during thyroglobulin secretion and resorption. Endocrinology 93 248-252. (doi:10.1210/endo-93-1-248)

Wright GM, Filosa MF \& Youson JH 1978 Immunocytochemical localization of thyroglobulin in the endostyle of the anadromous sea lamprey, Petromyzon marinus L. American Journal of Anatomy 152 263-268. (doi:10. 1002/aja.1001520207)

Wu H, Suzuki S, Sellitti DF, Doi SQ, Tanigawa K, Aizawa S, Akama T, Kawashima A, Mishima M, Ishii N et al. 2009 Expression of a thyroglobulin (Tg) variant in mouse kidney glomerulus. Biochemical and Biophysical Research Communications 389 269-273. (doi:10.1016/j.bbrc.2009.08.129)

Yi X, Yamamoto K, Shu L, Katoh R \& Kawaoi A 1997 Effects of propyithiouracil (PTU) administration on the synthesis and secretion of thyroglobulin in the rat thyroid gland: a quantitative immuno-electron microscopic study using immunogold technique. Endocrine Pathology $\mathbf{8}$ 315-325. (doi:10.1007/BF02739934)

Zheng G, Marino M, Zhao J \& McCluskey RT 1998 Megalin (gp330): a putative endocytic receptor for thyroglobulin (Tg). Endocrinology 139 1462-1465. (doi:10.1210/en.139.3.1462)

\section{Received in final form 14 February 2011 \\ Accepted 4 March 2011 \\ Made available online as an Accepted Preprint \\ 4 March 2011}

